\title{
British Food Journal: gaining global ground
}

\author{
Kerry Chipp, Raeesah Chohan, Caitlin Ferreira and Astrid Ringas
}

\section{Introduction}

This editorial reviews the publications in the British Food Journal (BFJ) over the past eleven years whilst under the stewardship of the present editor. During this time the journal has further advanced onto the international stage with its inclusion in the Science Citation Index (Expanded) and Current Contents (Agriculture, Biology and Environmental Sciences) in November 2005 (Emerald Publishing, 2012).

The journal has witnessed a large increase in submitted papers; increased its rejection rate and placed greater quality demands on its authors. The editor has made a concerted effort to be inclusive, increasing total publication rather than rejecting high quality papers due to space constraints (Griffith, 2015). The aims of this editorial are threefold: to review the international compass of research published under the current editor, perform additional analyses of methodological and research design issues, and to examine author productiveness, source and impact.

\section{Literature Review}

With growing pressure to evaluate research, a bibliometric citation analysis has emerged as a commonly employed tool (Bartoli and Medvet 2014) entailing the measurement of information behaviour and properties (Hussain and Fatima 2011). The British Food Journal has conducted a number of reviews in its long 116 year history (Mozley, 1994; Willis, 1987), most notably upon its centenary (Collins and Oddy 1998). All three of these reviews have been more qualitative than quantitative, with analyses of trends in food research at the forefront. The current editor, Chris Griffith, had the stated aim in 2003 to make the journal more global and so to encourage audiences to "look beyond the "British" in [the] title and see the breadth of [its] research"(Emerald Publishing 2012, p.1). He went on to add that he was "constantly looking at ways of furthering the journal's international research status" (Emerald Publishing 2012, p.1). Such sentiments echo his predecessor, Willis. Willis aimed to provide readers with information from other countries if such was of a better standard than that found in Britain (Willis, 1987). Thus it is both appropriate and timely for a more quantitative analysis of the journal with a particular view to demonstrating its international bona fides. It is also a good conduit to unpicking why, despite a growing plethora of publication outlets, submissions to the BFJ have increased substantially and consistently (Griffith, 2015).

Bibliometric analyses of food-related journals are evident in past literature and the British Food Journal has been captured in their ambit. Dias, Schultz, Schuster, Talamini and Révillion (2015) conducted an analysis of publications about the 'organic food market' from 1945 until 2013, quantitatively assessing the impact, methodological preferences, themes and international sources of studies in the area. Articles in the British Food Journal attained fifth 
and $15^{\text {th }}$ place in the resulting bibliometric assessment (Dias et al., 2015). The journal has also featured in country specific bibliometric studies, namely in India (Jeyshankar et al., 2014), attaining $15^{\text {th }}$ place in their empirically derived list of core journals in food and nutrition research in that country.

Journals in the field have moved to quantify impact, such as the Journal of Food Science and Technology from 2000 - 2004 (Vijay and Raghavan 2007). Such studies included reviews of contribution numbers, authorship patterns, and the geographical spread of researchers and the influence of developed and developing nations (Vijay and Raghavan 2007). Since the most recent review of the BFJ was completed in 1998 (Collins and Oddy 1998) and the current editor appointed in 2003, it is both timely and appropriate that such an analysis be conducted of the BFJ from 2004 to present.

This type of approach would help potential authors by widening the scope of journal assessment, from the narrow and at times obsessive focus on impact factors the limitations of which Griffith (2015) covers at length , to a carefully considered review of the journal submissions themselves. Griffith in his editorial (2015), by providing download and and other user metrics went some way towards this but the current editorial seeks to add to this with additional perspectives and data .

\section{Methodology}

Similar to previous bibliometric studies (for example, Coudounaris, Kvasova, Leonidou, Pitt, and Nel, 2009), we employed predefined criteria to select articles: firstly, time period: The current review incorporated all data from 128 issues published during the 10.5 year period 2004-2015, a period not hitherto covered, and spanning the tenure of the sitting editor. Second, manuscript type: only peer reviewed research articles, case studies, reviews etcetera were included, eliminating editorials, comments, book reviews and replies. All special issues were also included in the analysis.

Articles were initially sourced through Harzing's Publish or Perish as it allows for the easy extraction and tabulation of bibliometric characteristics from a set of articles extracted from Google Scholar (Jacsó, 2009). After the list was de-duplicated on title and article URL, 1110 articles remained. Further de-duplication on abstract reduced the pool further and manual coding of additional categories of the research discovered omissions from Publish or Perish. A final tally of 1033 articles was made after cross-checking the software-generated list with that of Emerald Publishing's database. Many papers were found to be undetected by the software and were thus coded manually and therefore had no GSRank. GSRank refers to the order in which Google returns the search results, with earlier ranks often indicating more relevant results (www.harzing.com). Correspondingly, these had no citation figures. Citation figures were then retrieved manually from Google Scholar which reduced the number of papers without citations to 192 or $18 \%$ and the data was recorded as zero rather than missing. Manual entry was only done for papers without citations; Harzing believes that Google Scholar could over estimate citations, but that it is superior to Thomson ISI Web of Science, as other authors have noted too (for example, Coudounaris et al., 2009). Thus, it was deemed 
best to restrict manual entry to the cases of missing data rather than the entire database. Sense checks of the coded data were undertaken through the use of logic formulae in Excel. Anomalies were identified and double checked thereafter. One article was found to be in BFJ but Google Scholar falsely identified it as sourced from the journal Nutrition and Food Science (Grewal and Jood 2009).

A coding protocol was enacted to facilitate a content analysis of, first, author demographics, location, number and resident institution and whether they were academics, practitioners or doctoral students; second, research design characteristics, which were categorised as being either quantitative or qualitative. In circumstances where both were used, both were acknowledged. The articles were then further coded by the more detailed categories of the research method employed. In cases where more than one type of research method was used, all methods were acknowledged and received an equal weighting.

The third topic considered the statistical techniques employed along a coding protocol developed by Botha et al. (2011). This allowed for all articles that made use of quantitative research to be subdivided into more diverse categories.

\section{Results}

\section{Authorship characteristics: an international pool}

While the average number of authors increased slightly across the ten year period under review, from 2.6 authors in 2004/07 to 2.9 in 2012/15, an analysis of the frequency data reveals that single authored papers have been on the decline from 23\% (in 2004-2007) to 14\% (2012-2015), a 9\% drop. Papers with multiple authors, particularly those with four or more, have increased ( $8 \%$ in 2004/07 to $14 \%$ in 2012/15) and have been of a progressively multinational nature. Ninety one percent of authors came from one country in 2004/07 while only $78 \%$ did in 2012/15. Authorships across three or more countries doubled between 2004 and 2015 , from $2 \%$ to $4 \%$. The total number of countries has not increased much on average (1.1 to 1.3). The contribution of researchers outside the West increased considerably, however: Western Europe stood at 70\%, North America 8\% and the Antipodes $11 \%$ in 2004/07. Ten years on, writers from the Middle East, Central and South America have both more than trebled ( $2 \%$ to $7 \%$ ), Asians have more than doubled (6\% to $16 \%$ ), and Eastern Europe has moved from zero in 2004/07 to 4\% in 2015. Western Europe has therefore decreased in total percentage quite substantially, from $70 \%$ to $57 \%$ - nearly a quarter. The years 2008 and 2010 witnessed the greatest sea change in global reach: in 2008, 21.4\% of authors came from emerging markets - up from $0 \%$ the previous year. This surge abated somewhat to $17.6 \%$ in 2009 only to bounce back higher to $28.6 \%$ in 2010 . 2015, with only half the years' articles in, has matched that highpoint, with $28.8 \%$ of authors coming from emerging markets.

Seventy six nations are represented in the journal across the time period under review, representing $39 \%$ of the globe. When country of origin is reviewed at a granular level, the BFJ has, over time, represented far fewer articles from the United Kingdom (103 in 2004/07 
to 69 in 2012/15). Australians and Americans have featured strongly, but, similar to the Dutch prominence in other management science journals, this country has increased its presence in BFJ - from 16 papers in 2004/07 to 26 in 2012/15. Other Western European national contributors, which have increased, are Italy, Germany and Spain. The remainder of the top ten is from Western Europe (Norway and Finland), apart from India, which places eighth. India, along with another other BRICS nation, Brazil (in $12^{\text {th }}$ place) has gone from a low base of 9 papers to 19, while Brazil has remarkably moved from 2 articles in 2004/07 to 21. Of the remaining nations of the BRICS, China, Russia and South Africa, China had strong growth, moving from 1 in 2004/07 to 16 in 2012/15. If Taiwan is counted with China, the People's Republic would have 49 articles and move into $6^{\text {th }}$ place. South Africa doubled its contribution from 5 papers in 2004/07 to 12, but its absolute number remained low, placing it in $21^{\text {st }}$ place. Russia was the poorest performer of the BRICS, standing in $48^{\text {th }}$ place with only two articles.

Other nations, with much increased involvement, are Turkey $\left(13^{\text {th }}\right)$, Sweden (5 to 20), Denmark (7 to 15 ), Malaysia (0 to 11 ), Poland (0 to 8), Pakistan (0 to 8), Portugal (0 to 7), Canada (6 to 14) and Switzerland (1 to 6). Nations, aside from the UK, with reduced contribution were Ireland, Greece, France and Nigeria.

The relationships between academics, multiple authorships and practitioners demonstrated that the greater the numbers of contributors, the more likely were these to span academia and practice. Very few sole authored articles were the domain of practitioners - only 23 articles in total, while academics produced 147 single authored papers. Academics were more likely to work in pairs (273 papers had two academic authors) and practitioners were more inclined to partner with academics than each other.

\section{Research characteristics}

Just as the journal has become more global over the last ten years, the quantitative nature of research published therein has increased nearly threefold - from 115 papers using quantitative methods in 2004/07 to 309 papers in 2012/15. Experiments, in particular, have gained ground, doubling from 30 to 68, while the use of surveys has increased from 75 in 2004/07 to 199 in 2012/15. Use of qualitative research, from constituting the majority of articles in $2004 / 07$, has fallen to a third of this figure in 2012/15. Analytics within quantitative studies have become more complex, with multivariate techniques gaining currency, particularly regression, factor and cluster analysis and structural equation modelling (SEM). Regression and factor analysis are the two most commonly used techniques, but with the increased prevalence of SEM software, training and use within academia, its increased deployment could be a sign of things to come. The nature of techniques used must be in line with the content; such quantification, therefore, could be demanded by the topics at hand, suggesting more complex models of areas where much is known, hence more quantitative studies.

When research characteristics are profiled by degree of national development, it appears that emerging markets are far more likely to rely on quantitative methods, as $63 \%$ to $19 \%$ of their work is quantitative rather than qualitative, as indicated in Table 1. Nevertheless, the quantitative work itself tends to be more univariate than multivariate (93\% to 55\%), with a 
reliance on means tests and ANOVA. Authors from the developed world also use descriptive and univariate tests $(87 \%)$, but these appear to be a precursor to more advanced analysis, as $73 \%$ proceeded to multivariate testing.

Table 1: Research Design, Methodology and Statistical Techniques According to Market Type Publication

The most prolific authors have some relationship with the most influential. Verbeke, Tikkanen, Mondelaers, Aertsens and Van Huylenbroeck feature in both lists. Three are Dutch and one is a Finn. The most prolific are Verbeke and Manning (UK), both on 16 papers apiece; Tikkanen is on 9, Mondelaers 7, Aertsens 6 and Van Huylenbroeck 6. The United Kingdom has eight of the twenty highest producers, followed by the Netherlands (7) and Australia (2). India has one strong practitioner contributor in S Sarkar (7) and Ireland has likewise in J Kennedy (5).

\section{Conclusion}

Sole authored papers have been on the decline, similar to other bibliometric studies (for example, Coudounaris et al., 2009). Multiple authorships have increased their international flavour, with a strong increase in multi-national collaboration, the nature of which is trending more towards work by authors based in both the developed- and developing worlds. True to Griffith's aim to make the journal more worldwide, authors now come from $39 \%$ of the globe. The BRICS nations, notably Brazil, India, China (if Taiwan is included) and South Africa have all increased their contribution. Other notable author nationalities are Dutch, Scandinavian and Turkish. Practitioners from the developing world are making their presence felt, with one (Sarkar) placing in the list of the most prolific.

A review of the methodology demonstrates a growing preference for quantitative studies across the board, although the sophistication thereof is skewed towards the developed world. Authors from the developed world also appear to be more at ease with qualitative methods as they are more likely to employ them than researchers from the developing world. The decline in qualitative approaches is in some ways both understandable, people feel happier and reassured with numbers and statistical values but is also sad. Well conducted qualitative studies can provide a greater understanding about the reasons why consumers behave the way they do and can now themselves be analysed more objectively

There is an overlap between productivity and impact for the Dutch authors Verbeke, Mondelaers, Aertsens and Van Huylenbroeck and the Finn, Tikkanen. Authors from the United Kingdom are more likely to be on the prolific list: Manning, Griffith, Baines and Jones along with Alonso (Australia) and Frewer (Netherlands). Authors from the developing world should take note of their methodologies and consider not only advanced quantitative methods but qualitative ones also. All researchers need to think carefully on practitioner and international collaboration as this is increasingly becoming effective. 
We were limited by the benefits and hazards of Publish or Perish and the dual manual checking and coding. It was noted that Google Scholar may return an over-estimation, thus our manual coding of citations for the missing data may be reflective of this. In terms of manual data entry, although various sense checks were employed and four coders used, researcher error cannot be eliminated completely.

There is empirical evidence that the BFJ has transcended the "British" in the title; the sheer scope of article sourcing and researcher collaboration truly makes the publication a global player. The publication has grown in reputation, quality and size (Griffith, 2015), no ordinary feat, while maintaining quality and encouraging research methods of increased complexity.

\section{References}

Bartoli, A. and Medvet, E. (2014), "Bibliometric Evaluation of Researchers in the Internet Age.”, Information Society, Vol. 30 No. 5, pp. 349-354.

Botha, E., Lilford, N. and Pitt, L. (2011), "South African management literature over the past fifteen years: Content analysis of the three top South African management journals", South African Journal of Business Management, Sabinet Online, Vol. 42 No. 4, pp. 8998.

Collins, E. and Oddy, D.J. (1998), "The centenary of the British Food Journal, 1899-1999changing issues in food safety regulation and nutrition", British Food Journal, MCB UP Ltd, Vol. 100 No. 10/11, pp. 433-550.

Coudounaris, P.C.D., Kvasova, P.C.O., Leonidou, L.C., Pitt, L.F. and Nel, S.L.D.D. (2009), "Fifteen Good Years", Management international review, Springer, Vol. 49 No. 5, pp. 671-684.

Dias, V. da V., Schultz, G., Schuster, M. da S., Talamini, E. and Révillion, J.P. (2015), “The organic food market: a quantitative and qualitative overview of international publications", Ambiente \& Sociedade, SciELO Brasil, Vol. 18 No. 1, pp. 155-174.

Emerald Publishing. (2012), "Meet the editor of... British Food Journal An interview with: Chris Griffith", Emerald Group Publishing, available at: http://www.emeraldgrouppublishing.com/authors/interviews/bfj.htm\#note (accessed 26 July 2015).

Grewal, A. and Jood, S. (2009), "Chemical composition and digestibility (in vitro) of green gram as affected by processing and cooking methods", Nutrition \& Food Science, Emerald Group Publishing Limited, Vol. 39 No. 4, pp. 342-349.

Griffith, C.J. (2015), “Editorial”, British Food Journal, Emerald, Vol. 117 No. 1, doi:10.1108/BFJ-10-2014-0360.

Hussain, A. and Fatima, N. (2011), "A bibliometric analysis of the 'Chinese Librarianship: an International Electronic Journal,(2006-2010)’”, Chinese Librarianship: an International electronic journal, Vol. 31. 
Jacsó, P. (2009), "Calculating the h-index and other bibliometric and scientometric indicators from Google Scholar with the Publish or Perish software", Online Information Review, Emerald Group Publishing Limited, Vol. 33 No. 6, pp. 1189-1200.

Jeyshankar, R., Rao, P.N. and Vellaichamy, A. (2014), "Mapping of Research Output of Food and Nutrition Literature in India.", International Journal of Information Dissemination \& Technology, Vol. 4 No. 1.

Mozley, D. (1994), "British Food Journal-A History”, British Food Journal, MCB UP Ltd, Vol. 96 No. 5/6, pp. 1-92.

Vijay, K.R. and Raghavan, I. (2007), "Journal of Food Science and Technology: a bibliometric study", Annals of Library and Information Studies, Vol. 54 No. 4, p. 207.

Willis, M. (1987), "British Food Journal-A Retrospective”, British Food Journal, MCB UP Ltd, Vol. 89 No. 6, pp. 130-139. 